\title{
ECG and PLI Artifacts Cancellation from Electromyogram
}

\author{
${ }^{1}$ Promila Kamboj, ${ }^{2}$ Simran Jit Singh \\ ${ }^{1,2}$ (ECE,SDDIE/ kurukshetra university kurukshetra , India)
}

\begin{abstract}
Electromyography (EMG) is a test used to discern primary muscle conditions from muscle weakness caused by neurological disorders. EMG recordings are frequently contaminated by noise. The objective of this research was to remove or reduce the two main noises which disturb the EMG signal with help of filtering. The most common noises which are present in the EMG signal are the electrocardiogram $(E C G)$ and the power line interference (PLI). The butter worth filter is proposed to cancel the ECG noise, and notch filter for removal of PLI artifacts from the main EMG signal and artificial neural network (ANN) which give the error signal (i.e. difference between the actual and target values) and enhance the signal-to-noise ratio of the output signal. The performance evaluation of the proposed technique is done in terms of signal-to-noise ratio, mean square error, and convergence time.
\end{abstract}

Keywords - Artificial neural network, Digital Filters, Electromyogram (EMG), ECG artifact, PLI.

\section{INTRODUCTION}

Electromyography (EMG) is a very powerful diagnostic test of human body. An electromyography detects the electrical potential generated by muscle cells when these cells are electrically or neurologically activated. EMG is used to distinguish primary muscle conditions from muscle weakness caused by neurological disorders. It is also used to find causes of weakness, paralysis, involuntary twitching and abnormal levels of muscle enzymes [5]. It helps to diagnose nerve damage and muscle damage. EMG is sometimes referred to as myoelectric activity. The signal is normally a function of time and is describable in terms of its amplitude, frequency and phase. Electrical impulses are shown as wavelike tracings on a cathode-ray oscilloscope and recorded as an electromyogram (EMG). Each biomedical signal has its own characteristics that differentiate it from other biomedical signal. The characteristics of the normal (without any disease) EMG signal are given below:

i. The Amplitude of EMG signal lies between: 0-10 mV.

ii. Dominant energy is in the $50-150 \mathrm{~Hz}$ range.

iii. The usable energy of the signal is limited to the 0 to $500 \mathrm{~Hz}$ frequency range.

iv. Bandwidth of the EMG signal is in the range of: $20-2000 \mathrm{~Hz}$.

\subsection{NoISES OR ARTIFACTS IN EMG}

In general, noise is defined as electrical signal that are not the part of the desired signal. The EMG signal depends on the anatomical and physiological properties of muscles. It acquires noise while traveling through different tissues. Due to the presence of different types of the noise which are occurring in the EMG signal (during the conduction of the EMG test) destroy the important information regarding the diseases. Biomedical signals are often contaminated by noise from sources such as power line interference and disturbances due to movement of recording electrodes. With the addition of these noises the biomedical signals are often interfere with one another, e.g., signals due to muscles contractions often contaminate electrode encephalograms (EEG's) and electrocardiograms (ECG's). ECG, which is frequently present when EMG is recorded from electrode sites near the trunk and neck area. These noises alter the real shape of the original EMG signal. For removing these noises digital filters are used. Therefore filters play an important role in the medical field for removing noise and interference. Noise removal is complicated process because the characteristics of almost all biomedical signals vary in time.

There are number of reasons, due to which noise signal is introduced in to the EMG signal. The EMG detector, if it is at the surface of the skin, collects signals from different motor units at a time which may generate interaction of different signals. A primary source of noise is the electrical or magnetic signals produced by nearby devices and power lines as human body behaved like an antenna which caught electromagnetic interference signals [14]. Moreover, inaccuracies in the sensors, poor contact between sensor and source (patient), and disturbances from signals produced by physiological processes. The artifacts are the superimposed signal that can hide some useful information in a measured signal. It is important to understand the characteristics of the electrical noise. The main task of processing biomedical signals is to filter the signal of interest out of from the noisy background and to reduce the redundant data stream to only a few, but relevant parameters. 
Then, several filtering techniques were developed to reduce the noises from the EMG signal in the past such as the low pass differential filter for the analysis of EMG signals is defined in [1]. High pass filter (HPF) is used in [2] to effectively remove ECG artifact from raw EMG and suggested that a cutoff frequency of approximately $30 \mathrm{~Hz}$ is desirable for balancing ECG removal with excessive EMG. A simple method for removing power line artifact from EMG in the time domain is suggested in [3]. Two different methods were compared for their efficacy in removing $50 \mathrm{~Hz}$ noise. The first was a simple second-order recursive digital notch filter and the second method called spectrum interpolation, compared in [4]. Back propagation network, explains the concept of adaptive interference cancellation and efficiency of BPN to cancel the ECG interference in EMG signal is given in [5]. The Real-time filtering technique can be used to remove ECG interference from recorded EMG. The gating technique came in to use before the Real-time filtering [6]. Elimination of Power Line Noise from EMG signals using an efficient adaptive laguerre filter is suggested in [7]. In this paper a novel algorithm is proposed for cancelling the Power Line Interference (PLI) and its harmonics from Electromyography (EMG) signals using an adaptive IIR Laguerre filter. A de-noising method using independent component analysis (ICA) and a high-pass filter to effectively suppress the interference of ECG in SEMG recorded from trunk muscles is discussed in [8]. ICA analysis was applied to separate SEMG from ECG artifacts based on the assumption that muscle and heart activities are anatomically and physiologically separated processes and their independency is reflected in the statistical relation between the electrical signals generated by those processes is addressed in [9]. Also the artifacts removal can be performed by using Discrete Wavelet (DWT) or Independent Component Analysis, in this paper [10], the author present a method based on the joint use of wavelet transform and Independent Component Analysis. Three methods for offline removal of power line interference from electromyograms were suggested in [11]. To remove the ECG from EMG signal, adaptive filtering technique is used, ANC filter based on a RLS algorithm can adaptively track the filter coefficients according to the input signals, and it should maintain similar performance when applied to a variety of muscle groups. This adaptive filter was effective and efficient for noise cancellation of surface EMGs [12]. In the next, the authors present three methods of artifact removal: (a) Wavelet filter (b) Neural ICA filter and (c) the mixed Wavelet-ICA filter are addressed in [13]. To remove two noises which disturb the surface electromyography signal that is: ECG and PLI a new Lean Mean Square (LMS) Widrow adaptive structure is proposed in [14]. RBF neural network as compared with other types of neural networks can be effectively remove the noise from EMG is explained in [15]. A new method is designed based on filtering of EMG signal corrupted with interference of power line and ECG (EMG+PLI+ECG), by using Matching Pursuit (MP) is given in [16].

\section{NOISE REMOVAL METHODS}

It become necessary to reduce or minimize the noise from the EMG test signals so that doctor can easily diagnose the problem or diseases.

ECG signal (act as noise in EMG signal) removal from EMG signal: The electrocardiogram (ECG) is the recording of the heart's electrical potential versus time. The ECG is the most common noise which is present in the EMG signal when this signals taken from the trunk and neck side. Several methods are proposed to minimize the ECG interference from the EMG. Among these the adaptive method is very efficient method for removing ECG interference. Here the butter worth filter with the combination of Artificial Neural Network (adaptive method) is used. This filter is used for its maximally flat response in the transmission pass band, minimizing pass band ripple. This filter is best suited for applications requiring preservation of amplitude linearity in the pass band region, this feature which makes the Butterworth filter an ideal candidate for conditioning the EMG signal. The cut off frequency is selected based on the frequency component of the estimated signal. The order of the filter is two, and the cutoff frequency $W_{n}$ is 0.1 .The filter type is high pass filter.

Power Line Interference removal from EMG signal: A major problem in the recording of EMG test is that the measured signal is corrupted by $50 \mathrm{~Hz}$ power line interference. Many methods were proposed in the past for the removal of power line interference. It occurs in the middle of the EMG spectrum and comes from both electric field and AC power lines. Notch filtering is very powerful method which is used to remove the power line noise. This filter is very important in a wide variety of instrumentation applications, from telecommunications to biomedical signals processing, where often it is necessary to remove a narrow band or even a single frequency of the measurement signal. It is so far known that IIR adaptive notch filter realization performs better than finite impulse response (FIR) counterparts as regards the number of coefficients and computational complexity. A notch filter removes a particular frequency from a signal and has a frequency response that falls to zero over a narrow range of frequencies (i.e. a $50 \mathrm{~Hz}$ notch may block signals from 49.5 $50.5 \mathrm{~Hz})$. 


\section{DESIGN OF Proposed Model}

As described earlier that objective of this work is to design a new technique to remove the artifacts from the EMG signal so that physician gets good quality of EMG signal. In order to full fill this objective, the number of task should be performed and work procedure to be adopted for this proposed work is as follows:

Step:1 Download the data (EMG data) from the Physio.net of all the subject. The data should be Matlab compatible i.e. data.mat file.

Step:2 Load the signal in Matlab.

Step:3 Add synnthetic Power line noise to the signal.

Step:4 Add ECG noise to the main EMG signal.

Step:5 Calculate the measured signal.

Step:6 Remove the unwanted signal or the charcteristics from the signal using Notch filter. Notch filter is used to remove the power line noise from the EMG signal. The frequency range of the power line is lies between 50 to $60 \mathrm{~Hz}$. The ECG noise is remove using Butter worth filter.

Step:7 Take the signal from the previous step and apply it to the Artificial Neural Network. The ANN is work as the adaptive filter.

A training pair is presented to the network. Neurons in each layer calculate the net value. At the output layer, get the error signal which is the difference between the actual and target values. By adjusting the weight values of the neurons in each layer the error can be minimized or so this is called the supervised learning. The algorithm which is used to weight updating is the gradient descent or back propagation algorithm. The speed and accuracy of the learning process depends on the learning rate. There are two phases of data flow. First, the input pattern is propagated from the input layer to the output layer and, as a result of this forward flow of data, it produces an actual output. Then the error signal is back propagated from the output layer to the previous layers to update their weights.

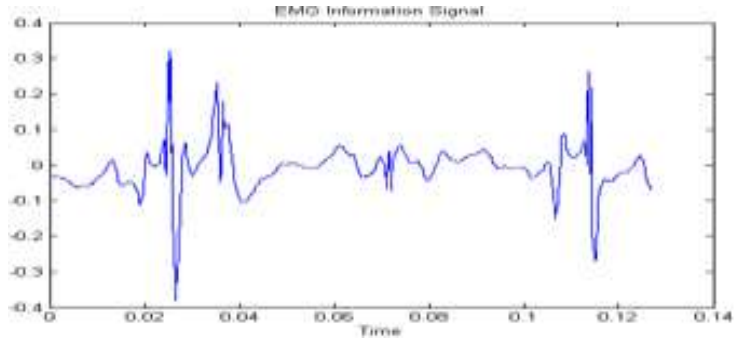

Figure 1 EMG signal

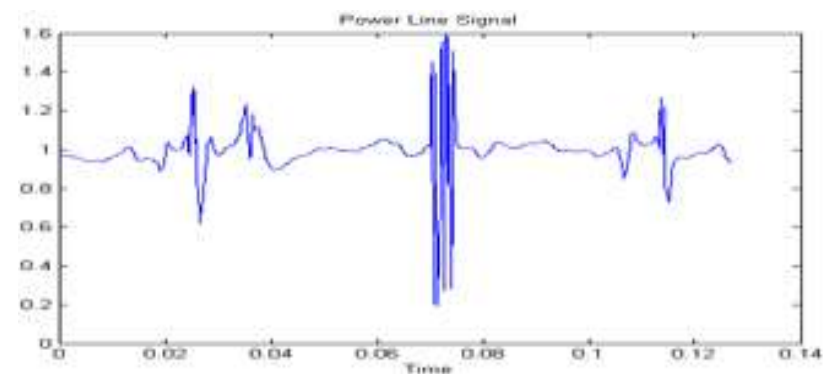

Figure 2 Power line signal

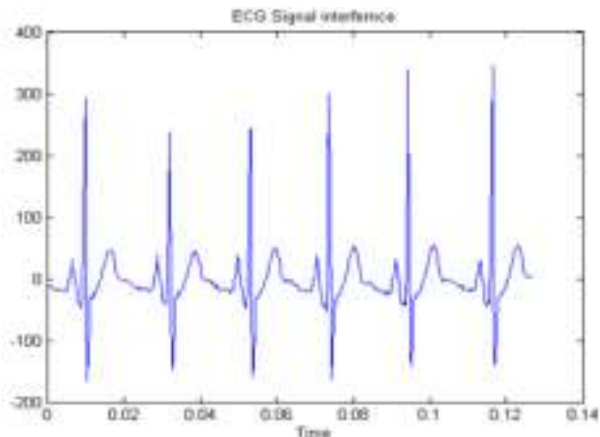

Figure 3 ECG signal

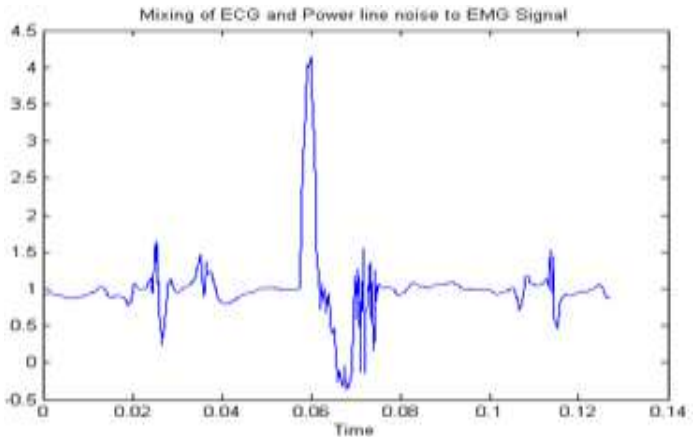

Figure 4 Mixing of ECG and PLI to EMG signal 
"Fig.1" shows the EMG signal of a healthy person. Hence EMG signal is a complicated signal, which is calculated by the nervous system and is dependent on the anatomical and physiological properties of muscles. This is a waveform of 44 year old man without history of neuromuscular disease. "Fig. 2" shows the Power Line signal which acts as the noise in the main EMG signal and "Fig. 3" shows the ECG signal which is also act as the noise and the "Fig.4" shows the mixing of the two noises with the main EMG signal. In these graphs, the " $\mathrm{x}$ " axis represents the time and " $y$ " axis represents the amplitude value of the signal.

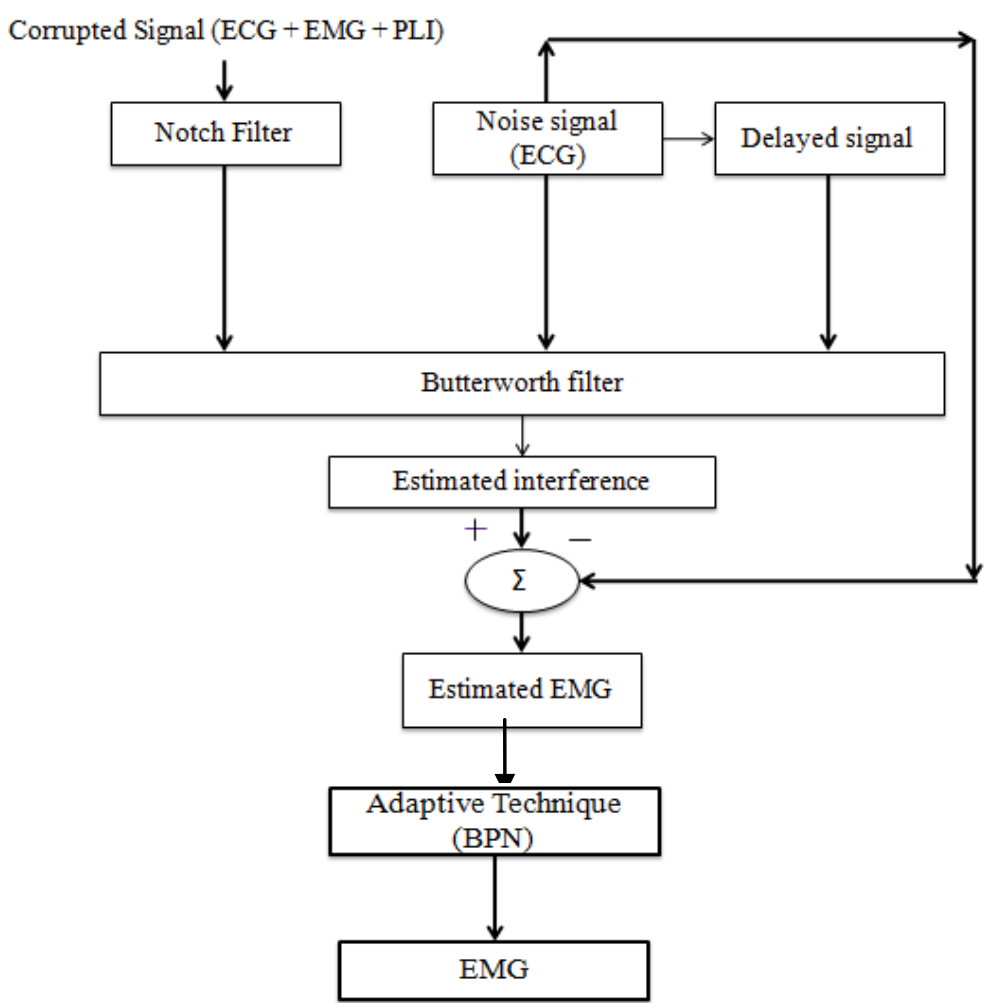

Figure 5 Flowchart for the proposed implementation of Notch filter and AIC

\section{Simulation AND EXPERIMENTAl RESUlts}

It is desirable to obtain an EMG signal that contains the maximum amount of information and minimum amount of contamination. Thus, the maximization of the signal- to-noise ratio should be done with minimal distortion to the EMG signal. Here the results obtained from the proposed method are shown below:

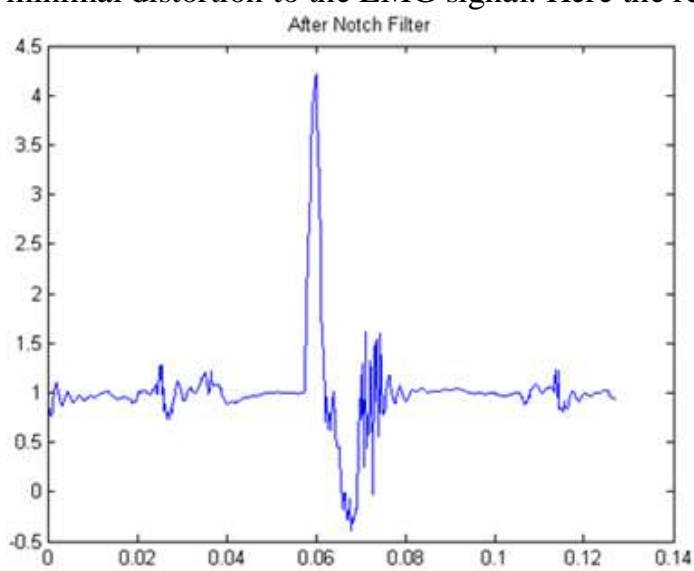

Figure 6 EMG signal after apply the Notch filter

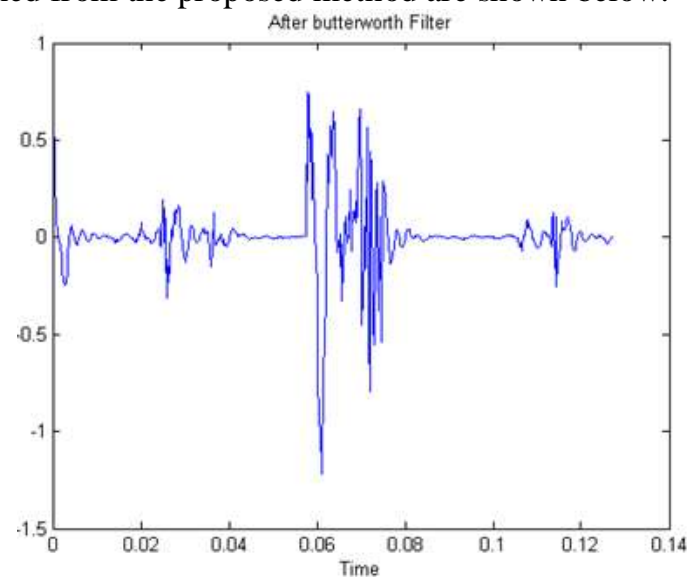

Figure 7 Noise free EMG signal after applying Butterworth filter

The known ECG and PLI signals are given to the Notch filter as shown in the 'Fig.5'. Here the PLI noise is removed/ reduce and get the PLI free EMG signal as shown in 'Fig. 6', the rest part of the signal i.e. ECG + EMG and the delayed ECG signal are given as an input to the butterworth filter. From the butterworth filter get the estimated EMG signal as given in 'Fig. 7', and further fed to the ANN. Difference between the 
three signal (i.e. Input, Output and Target signal) is demonstrated in 'Fig. 8'. In the ANN the BPN algorithm is used to get the pure EMG signal and the performance graph is obtained as shown in 'Fig. 9'. All this programming has been done in the Matlab.

For this, ANN is designed with the following specifications:

i. $\quad$ The input to the neural network is EMG signal of a healthy person.

ii. In the input layer and the output layer, the number of neuron is one.

iii. In the hidden layer the number of neurons is 10.

iv. The activation function for the input layer is the TANSIG, and for the hidden layer the activation function is PURELIN.

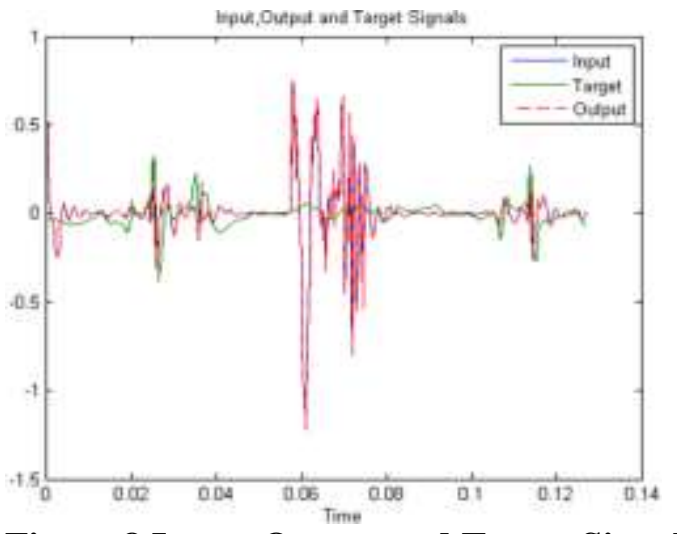

Figure 8 Input, Output and Target Signal



Figure 9 Training result of BPN

TABLE 1.PERFORMANCE EVALUATION

\begin{tabular}{|c|c|c|c|c|}
\hline S. No. & Epochs & $\begin{array}{c}\text { Mean Square } \\
\text { error }\end{array}$ & SNR (dB) & $\begin{array}{c}\text { Convergence Time } \\
\text { (sec.) }\end{array}$ \\
\hline 1 & 35 & 0.003709 & 17.8905 & 0.05 \\
\hline 2 & 54 & 0.003707 & 17.8886 & 0.04 \\
\hline 3 & 6 & 0.11272 & 17.9088 & 0.01 \\
\hline
\end{tabular}

\section{V.}

\section{CONCLUSION}

In this research work the entire focus was to get an estimated signal which is free from the ECG and Power Line interference. The presence of artifacts in real signal can cause hindrance in its interpretation. It becomes an important task to preprocess the signal in order to keep the noise minimum. Since the Notch filter is best suited for handling noises occur having characteristics of sudden increase in a peak of signal, and for this the first step was to remove this interference by using Notch filter. After applying Notch filter, it was observed that certain unknown / un required characteristics are still left in the signal. Since although the level of nonlinearities of the estimated signal obtained from the Notch filter had less level of noise now, but the signal still required some filtering. So therefore Butterworth filter has been used and estimated signal is obtained from Butterworth filter. Now to automate this process, machine algorithm called BPNN (Back propagation neural network) is used and good results are obtained. In this research work the main motive was to get minimum square error close to zero so that the trained network has minimum possible unknown characteristics. The MSE (mean square error) started reducing but the conversion time increased little bit.

\section{References}

[1] S. Usui and I. Amidor, Digital low-pass differentiation for biological signal processing", IEEE Trans. on Biomedical Engineering, Vol. 29, No. 10, pp. 686-693, 1982.

[2] M.S Redfern, R.E. Hughe and D.B Chaffin, High-pass filtering to remove electrocardiographic interference from torso EMG recordings, Journal of Clinical Biomechanics, Vol.8,No.1, pp. 44-48, 1993.

[3] R.V Baratta, M. Solomonow, B.H Zhou and M. Zhu, Methods to reduce the variability of EMG power spectrum estimates, Journal of Electromyography and Kinesiology, Vol.8, No.5, pp. 279-85, 1998.

[4] D.T. Mewett, K.J. Reynolds, H. Nazeran, Reducing power line interference in digitised electromyogramrecordings by spectrum interpolation, Med. Biol. Eng. Comput. 42 (2004) 524-531.

[5] C. Kezi Selva Vijila, C.Ebbie Selva Kumar, Cancellation of ECG in Electromyogram Using Back Propagation Network, International Conference on Advances in Recent Technologies in Communication and Computing, pp. 630-634, 2009.

[6] Bassam Rhou, Mohamad Sawan, Tommy Desilets and François Bellemare, Real-time filtering technique to remove ECG interference from recorded EMG, IEEE Trans. on Biomedical Circuits and System, pp. 21-24, 2008.

[7] Mojtaba Malboubi, Farbod Razzazi, Mahdi Aliyari. Sh, Elimination of Power Line Noise from EMG Signals Using an Efficient Adaptive Laguerre Filter, ICSEs Trans. on Signals and Electronic Systems, pp. 49-52, 2010. 
[8] Yong Hu, XH Li, XB Xie, LY Pang, Yuzhen Cao ,KDK Luk, Applying Independent Component Analysis on ECG Cancellation Technique for the Surface Recording of Trunk Electromyography, IEEE Trans. on Engineering in Medicine and Bilology Society, pp. 646-649, 2007.

[9] Joseph N.F.Mak, Yong Hu, KDK Luk, ICA-based ECG removal from Surface Electromyography and its effect on Low Back Pain Assessment, IEEE Trans. on conference on Neural Engineering, pp. 646-649, 2007.

[10] B. Azzerboni, M. Carpentieri, F. La Foresta and F.C Morabito, Neural-ICA and wavelet transform for artifacts removal in surface EMG, IEEE International Joint Conference on Neural Networks, Vol. 4, pp. 3223-3228, 2004.

[11] David T. Mewett, Homer Nazeran and Karen J. Reynolds, Removing Power Line Noise From Recorded EMG, IEEE Trans. on Engineering in Medicine and Bilology Society, Vol.3, pp. 2190-2193, 2001.

[12] Guohua Lu,John-Stuart Brittain, Peter Holland,John Yianni, Alexander. Green, John.Steina, Tipu, Aziza, Shouyan Wanga, Removing ECG noise from surface EMG signals using adaptive filtering, Elsevier Trans. On Biomedical Signals, pp 14-19, 2009.

[13] Ipsale Maurizio, Bramanti Alessia, D’Aleo Giangaetano, Azzerboni Bruno, Artifact Removal on surface EMG, Anno Accademico, Vol. XCIV, pp. 1-9, 2006.

[14] Slim Yacoub, Kosai Raoof, Noise Removal from Surface Respiratory EMG Signal, International Journal Trans. on Signal Processing, pp. 227-234, 2008.

[15] V. R. Mankar, Dr. A. A. G, Use of RBF neural network in EMG signal, WSEAS Trans. on Circuits and systems, Vol. 7, pp. 259265, 2008.

[16] Parisa Akbary, Hossein Rabbani, Removing power line interference and ecg signal from EMG signal using matching pursuit, IEEE 10th International Conference on Signal Processing (ICSP), pp. 1741-1717, 2010.

[17] Jancovic, M.M, Popovic, D.B., An EMG system for studying motor control strategies and fatigue, 10th Symposium on Neural Network Applications in Electrical Engineering, pp. 1-15, 2010.

[18] Parker,P., Merletti,R, Basic Physiology and Biophysics of EMG Signal Generation, IEEE Trans. on Electromyography: Physiology, Engineering, and Non-Invasive Applications, pp.1 -25, 2005.

[19] Hongying Meng, Alan Forster, and David Holder, Singularity Characteristics of Needle EMG IP Signals, IEEE Trans. on Biomedical Engineering, Vol. 53, pp. 219-225, Feb. 2006

[20] J. D. Costa Junior, D. D. Ferreira, J. Nadal and A. M. F. L. Miranda de Sa, Reducing electrocardiographic artifacts from electromyogram signals with Independent Component Analysis, IEEE Trans. on Engineering in Medicine and Biology Society (EMBC), pp. 4598-4601, 2010. 